Project ID Number 55032

\title{
Environmental Analysis of Endocrine Disrupting Effects from Hydrocarbon Contaminants in the Ecosystem
}

\author{
January 31, 2000 \\ Dr. John A. McLachlan \\ Tulane University \\ 1430 Tulane Avenue \\ New Orleans, Louisiana 70112 \\ Phone: 504-585-6910 \\ E-mail: jmclach@mailhost.tcs.tulane.edu
}

Six graduate students actively involved in research.

\section{RESEARCH OBJECTIVE}

This annual report summarizes the progress of three years of a three-year grant awarded to the Center for Bioenvironmental Research (CBR) at Tulane and Xavier Universities.

The objective of this project is to determine how environmental contaminants, namely hydrocarbons, can act as hormones or anti-hormones in different species present in aquatic ecosystems. The three major areas of research include 1) a biotechnology based screening system to identify potential hormone mimics and antagonists; 2) an animal screening system to identify biomarkers of endocrine effects; and 3) a literature review to identify compounds at various DOE sites that are potential endocrine disruptors. Species of particular focus in this study are those which can serve as sentinel species (e.g., amphibians) and, thus, provide early warning signals for more widespread impacts on an ecosystem and its wildlife and human inhabitants. The focus of the literature research was to provide an analysis of the contaminants located on or around various Department of Energy (DOE) sites that are or have the potential to function as endocrine disruptors and to correlate the need for studying endocrine disruptors to DOE's programmatic needs.

Previous research within the Center for Bioenvironmental Research at Tulane and Xavier Universities has focused on understanding the effects of environmental agents on the human and wildlife health and disease. In particular this research has focused on how exogenous agents can function to mimic or disrupt normal endocrine signaling, i.e. estrogen, thyroid within various systems from whole animal studies with fish, amphibians and insects to human cancer cell lines. Significant work has focused on the estrogenic and anti-estrogenic action of both synthetic organochlorine chemicals and naturally produced phytochemicals. Recent projects have extended these research objectives to examination of these environmental agents on the symbiotic relationship between nitrogen fixing rhizobial bacteria and leguminous plants.

This research will form the foundation for future experiments into the genetic manipulation of plants to potentially promote greater or more specific symbiotic relationships between plant and Rhizobium allowing this biological phenomenon to be used in a greater number of crop types. Future technology developments could include the genetic engineering of crops suitable for in situ vadose zone 
bioremediation (via microbes) and phytoremediation (through the crop, itself) in contaminated DOE sites.

\section{RESEARCH PROGRESS AND IMPLICATIONS}

As of the completion of this research, the CBR represents the Environmental Management Science Program's only research on the impacts of environmental endocrine disrupting chemicals. The CBR has over 5 years of expertise in developing model aquatic ecosystems for evaluating environmental problems relevant to DOE cleanup activities. CBR can elucidate (using biotechnology screens and biomarkers of exposure) how chemicals in the environment can signal the development of species in aquatic ecosystems, as well as, show detrimental impacts on community, population, and entire ecosystem levels, including humans. Since environmental endocrine disruptors are effective at concentrations similar to hormones, and can cause havoc at levels a thousand or more times below measurable levels of most current analytical methods, it is imperative to develop methods that are effective at concentrations in the environment. Therefore, research into low levels of environmental contaminants may show that currently accepted cleanup standards are inappropriate for DOE's cleanup efforts.

\section{RESEARCH RESULTS}

\section{Biotechnology Screening System (In vitro studies)}

The objective of this research was to determine the ability of PAHs identified in Louisiana's Bayou Trepagnier to function as hormone mimics or antagonists at environmentally relevant concentrations. Three biotechnology screens were developed and tested: 1) the Yeast Expression System (YES), 2) an in vitro screen for thyroid hormone disruption, and 3) an in vitro screen for the Arthropod ecdysone receptor. These screens allow us to test the ability of PAHs and other environmental hormones or hormone receptors of humans, amphibians, and invertebrates (e.g., shrimp). Yeast genetically engineered to produce human estrogen, progesterone, glucocorticoid, or thyroid hormone receptors were exposed to polyaromatic hydrocarbons known to occur in Bayou Trepagnier. The ability of the chemicals to interact with hormone receptors was determined by colormetric assay. Development of all of these screens is completed and all have demonstrated success in identifying numerous compounds, including PAHs, which interact with these important receptors.

\section{Animal Screening System (In vivo studies)}

Three animal screen studies have been funded through this grant and include the effects of environmental hormones on: 1) the African clawed frog (Xenopus laevis), 2) the leopard frog (Rana pipiens), and grass shrimp (Palaeomonetes spp.). Frogs were chosen as species of concern since unusual frequencies of frogs with morphological abnormalities have been observed recently in western North America; environmental hormones are capable of disrupting reproductive physiology in amphibians; amphibians are often the most susceptible species for adverse environmental impacts (and, thus, can serve as sentinel species for ecosystem inhabitants); and many are threatened and endangered. Grass shrimp were chosen as species of concern because of their economic and ecological value and because of the many industrial impacts on their aquatic habitats.

\section{In Vitro Kinetic and Thermodynamic Studies}

Kinetic exclusion assays were developed on the KinExA ${ }^{\mathrm{TM}}$ immunoassay instrument to quantify the thermodynamics and kinetics of binding reactions between the human estrogen receptor and individual 
compounds that compete with 17- $\beta$-estradiol for the hormone binding site on the receptor. Three different covalent conjugates of $17-\beta$-estradiol with bovine serum albumin (BSA) were surveyed for their ability to capture and permit the quantification of free, unliganded estrogen receptor in the KinExA: the 17-hemisuccinate; the 6-(O-carboxymethyl)oxime; and the 3-(O-carboxymethyl)oxime derivatives. Each $\beta$-estradiol BSA conjugate was adsorption coated onto polymethylmethacrylate beads and deposited in a packed bed above the porous screen in the observation/flow ceil of the spectrofluorimeter. The level of fluorescence observed from the beadpack was used to quantify the number of receptor molecules captured from the solution. Standard curves of instrument response as a function of the estrogen receptor concentration were linear up to $10 \mathrm{nM}$.

\section{Literature Review on DOE Site Contaminants}

The focus of this research is to provide analysis of the contaminants located on or around DOE sites have the potential to function as endocrine disruptors and to correlate the need for studying endocrine disruptors to DOE's programmatic needs. Extensive literature research was performed on: DOE/EM Complex cleanup needs and progress; U.S. Environmental Protection Agency (EPA) programs affecting DOE/EM; Environmental Risk Analysis and Management; and Community/stakeholder issues surrounding cleanup at the DOE complex.

\section{INFORMATION ACCESS}

L. Jin, D.Q. Tran, C.F. Ide, J.A. McLachlan, and S.F. Arnold. 1997. Several synthetic chemicals inhibit progesterone receptor-mediated transactivation in yeast. Biochemical and Biophysical Research Communications 233:139-146.

D.Q. Tran, C.F. Ide, J.A. McLachlan, and S.F. Arnold. 1997. The anti-estrogenic activity of selected polynuclear aromatic hydrocarbons in yeast expressing human estrogen receptor. Biochemical and Biophysical Research Communications 229:102-108.

A. Oliver Cheek, P.M. Vonier, E. Oberdörster, B. Collins Burow, and J.A. McLaclan. 1998. Environmental signaling: A biological context for endocrine disruption. Environmental Health Perspectives. 106. Supplement 1:5-10.

A. Oliver Cheek and J.A. McLachlan. 1998. Environmental hormones and the male reproductive system. Journal of Andrology. 19:5-10.

E. Oberdörster, and J.A. McLachlan. 1998. Interaction of PAHs, PCBs and phytoestrogens with the Drosophila Ecdysone Receptor in vitro. Toxicologist. 37(1):239.

A. Oliver Cheek, C.F. Ide, J.E. Bollinger, C. Rider and J.A. McLachlan. 1999. Alteration of leopard frog (Rana pipiens) metamorphosis by the herbicide acetochlor. Archives of Environmental Contamination and Toxicology. 37:70-77.

A. Oliver Cheek, K. Kow, J. Chen, and J.A. McLachlan. 1999. Potential mechanisms of thyroid disruption: Interaction of organochlorine compounds with human thyroid receptor $\beta 1$, thyroid hormone binding globulin, and transthyretin. Environmental Health Perspectives. 107:273-278.

E. Oberdörster, MA Clay and JA McLachlan, 1999. Inhibition of ecdysone-dependent gene-expression by phytochemicals. Toxicologist 48(1S):378.

L.G. Parks, A. Oliver Cheek, N.D. Denslow, S.A. Heppell, J.A. McLachlan, G.A. LeBlanc, and C.V. Sullivan. 1999. Fathead minnow (Pimephales promelas) vitellogenin: Purification, characterization and quantitative immunoassay for the detection of estrogenic compounds. Comparative Biochemistry and Physiology. 123:113-125.

E. Oberdörster, M Martin, CF Ide, J.A. McLachlan. 1999 Benthic community structure and biomarker induction in an estuarine system. Archives of Environmental Contamination and Toxicology. 37(4):in press. 
E. Oberdörster, DM Cottam, FA Wilmot, MJ Milner and JA McLachlan (1999) Interaction of PAHs and PCBs with Ecdysone Receptor using a multi-tiered approach. Tox. Appl. Pharm. 157:in press

E. Oberdörster, M Brouwer, T Hoexum-Brouwer, S Manning, and JA McLachlan. (in preparation) Inhibition of reproduction and molting by pyrene in the grass shrimp, Palaomonetes pugio. Environ. Health Persp.

\section{PRESENTATIONS}

Decline of Macro- and Meio-faunal Communities in Bayou Trepagnier, LA. E Oberdörster. presented at University of New Orleans, Mississippi River Water Quality Conference: Clean Enough? New Orleans, LA. September 21, 1997.

Effects of environmental chemicals on thyroid hormone induced metamorphosis in leopard frogs (Rana pipiens). A. Oliver Cheek, C.F. Ide, C. Rider, and J.A. McLachlan. presented at the Society of Environmental Toxicology and Chemistry annual meeting, November, 1997.

Multi-Tiered Approach to Detecting PAH Interactions with the Arthropod Ecdysone Receptor. E. Oberdörster, and J. A. McLachlan. presented at Gordon Research Conference on Environmental Endocrine Disruptors. Plymouth, NH. July 12-15, 1998.

Morphological and biochemical effects of environmental estrogens on frog (Xenopus laevis) metamorphosis. A. Oliver Cheek, C.V. Rider, M.A. Holstein, and J.A. McLachlan. presented at the Society of Environmental Toxicology and Chemistry annual meeting, November, 1998.

Potential mechanisms of thyroid disruption in humans: Interaction of organochlorine compounds with thyroid receptor, transthyretin, and thyroid binding globulin. A. Oliver Cheek, J. Chen, K. Y. Kow, and J. A. McLachlan. presented at the annual meeting of the Society of Integrative and Comparative Biology, January, 1999.

Bench to Bayou: Progress on Reproductive Disruption in Fishes. presented at the US-Japan Workshop on Endocrine Disruption. A. Oliver Cheek, T. Hoexum-Brouwer, S. Gronen, M. Brouwer, and J. A. McLachlan. Tulane University, New Orleans, LA, February, 1999.

Developmental exposure to anthracene and estradiol alters sex differentiation and reproductive success in medaka (Oryzias latipes). A. Oliver Cheek, T. Hoexum-Brouwer, S. Gronen, M. Brouwer, and J.A. McLachlan. To be presented at the Society of Environmental Toxicology and Chemistry annual meeting in November, 1999.

Developmental exposure to anthracene and estradiol alters reproductive success in medaka (Oryzias latipes). A. Oliver Cheek, T. Hoexum-Brouwer, S. Gronen, M. Brouwer, and J.A. McLachlan. To be presented at the Society of Integrative and Comparative Biology meeting in January, 2000. 